\title{
Community-based adherence clubs for postpartum women on antiretroviral therapy (ART) in Cape Town, South Africa: a pilot study
}

Allison Zerbe ${ }^{1 *}$, Kirsty Brittain ${ }^{2,3}$, Tamsin K. Phillips ${ }^{2,3}$, Victoria O. Iyun ${ }^{2,3}$, Joanna Allerton ${ }^{2,3}$, Andile Nofemela ${ }^{2,3^{\wedge}}$, Cathy D. Kalombo ${ }^{4}$, Landon Myer ${ }^{2,3}$ and Elaine J. Abrams ${ }^{1,5}$

\begin{abstract}
Background: With an increasing number of countries implementing Option B+ guidelines of lifelong antiretroviral therapy (ART) for all pregnant and breastfeeding women, there is urgent need to identify effective approaches for retaining this growing and highly vulnerable population in ART care.

Methods: Newly postpartum, breastfeeding women who initiated ART in pregnancy and met eligibility criteria were enrolled, and offered the choice of two options for postpartum ART care: (i) referral to existing network of community-based adherence clubs or (ii) referral to local primary health care clinic (PHC). Women were followed at study measurement visits conducted separately from either service. Primary outcome was a composite endpoint of retention in ART services and viral suppression $\mathrm{VS}<50$ copies $/ \mathrm{mL}$ based on viral load (VL) testing at measurement visits] at 12 months postpartum. Outcomes were compared across postpartum services using chi-square, Fisher's exact tests and Poisson regression models. The primary outcome was compared across services where women were receiving care at 12 months postpartum in exploratory analyses.
\end{abstract}

Results: Between February and September 2015, 129 women (median age: 28.9 years; median time postpartum: 10 days) were enrolled with 65\% opting to receive postpartum HIV care through an adherence club. Among 110 women retained at study measurement visits, 91 (83\%) achieved the composite endpoint, with no difference between those who originally chose clubs versus those who chose PHC services. Movement from an adherence club to PHC services was common: 31\% of women who originally chose clubs and were engaged in care at 12 months postpartum were attending a PHC service. Further, levels of VS differed significantly by where women were accessing ART care at 12 months postpartum, regardless of initial choice: $98 \%$ of women receiving care in an adherence club and $76 \%$ receiving care at PHC had VS $<50$ copies $/ \mathrm{mL}$ at 12 months postpartum $(p=0.001)$.

(Continued on next page)

\footnotetext{
*Correspondence: az2258@cumc.columbia.edu

Andile Nofemela is deceased.

'ICAP, Mailman School of Public Health, Columbia University, 722 W. 168th street, 13th floor, New York 10032, USA

Full list of author information is available at the end of the article
}

(C) The Author(s). 2020 Open Access This article is licensed under a Creative Commons Attribution 4.0 International License, which permits use, sharing, adaptation, distribution and reproduction in any medium or format, as long as you give appropriate credit to the original author(s) and the source, provide a link to the Creative Commons licence, and indicate if changes were made. The images or other third party material in this article are included in the article's Creative Commons. licence, unless indicated otherwise in a credit line to the material. If material is not included in the article's Creative Commons licence and your intended use is not permitted by statutory regulation or exceeds the permitted use, you will need to obtain permission directly from the copyright holder. To view a copy of this licence, visit http://creativecommons.org/licenses/by/4.0/ The Creative Commons Public Domain Dedication waiver (http://creativecommons.org/publicdomain/zero/1.0/) applies to the data made available in this article, unless otherwise stated in a credit line to the data. 
(Continued from previous page)

Conclusion: This study found comparable outcomes related to retention and VS at 12 months postpartum between women choosing adherence clubs and those choosing PHC. However, movement between postpartum services among those who originally chose adherence clubs was common, with poorer VS outcomes among women leaving clubs and returning to PHC services.

Trial registration: ClinicalTrials.gov NCT02417675, April 16, 2015 (retrospectively registered).

Keywords: HIV, Differentiated care, Postpartum, Retention in care, Adherence clubs, Maternal and child health

\section{Background}

Shifting guidelines away from CD4-guided antiretroviral therapy (ART) eligibility (Option A) to universal ART for HIV-infected pregnant and postpartum women (Option $\mathrm{B}+$ ) has led to a significant increase in the number of women on ART during pregnancy and breastfeeding [1]. While the Option B+ approach offers substantial benefits for the prevention of mother-to-child transmission (PMTCT) and maternal and child health $(\mathrm{MCH})$, concerns related to suboptimal maternal ART adherence and retention in HIV care have been well-documented [2-5]. While a study comparing CD4-guided ART eligibility to Option B+ in Eswatini did find improved retention at 6 months postpartum among women accessing PMTCT services under Option $\mathrm{B}+$, the authors noted that retention, specifically postnatally, was low across both approaches [6]. In a recent meta-analysis, retention rates among women enrolled in Option $\mathrm{B}+$ programs were found to be below those of the general adult population engaged in ART care [7]. Further, all studies included reported increasing loss to follow-up over time, highlighting specific concerns related to retention beyond the immediate postpartum period. With an increasing number of countries implementing the Option $\mathrm{B}+$ strategy and in turn managing a growing number of pregnant and postpartum women on ART, there is an urgent and critical need to identify effective approaches to retaining this highly vulnerable population.

Outside of $\mathrm{MCH}$ services, exciting and innovative ART service delivery models have been rolled out, specifically for stable adult patients established on ART [810]. Endorsed by the World Health Organization (WHO) in their 2016 guidelines [11], these cost-effective differentiated service delivery (DSD) models may lead to higher rates of retention and adherence among stable patients, while decongesting overburdened health systems [12-16]. In Cape Town, South Africa, a network of community-based adherence clubs has evolved since 2012 and now offers HIV care to over 32,000 ART patients (25\% of all ART patients) across the Cape Town health district $[17,18]$. To be eligible for the club system, patients must be non-pregnant adults, on ART for 6-12 months, with a suppressed viral load (VL) and no adherence issues or comorbidities that require ongoing clinical management. In this setting, high levels of retention and viral suppression among adults who are attending clubs have been reported [18].

Despite progress in rolling out various DSD models in Cape Town and other locations, questions remain about their potential among pregnant and postpartum women. To date, these models, including adherence clubs in the Cape Town health district, have focused primarily on stable patients established on ART with a suppressed VL and have actively excluded other key and vulnerable populations including pregnant and breastfeeding women [19]. However, recent advocacy by the WHO and others has encouraged expansion of these models to pregnant and postpartum women, recognizing that with the roll-out of Option $\mathrm{B}+$, there are increasing numbers of pregnant women presenting for antenatal care with known HIV status and who are stable on ART as well as large groups of women initiating ART in pregnancy $[19,20]$. With this in mind, we conducted a pilot evaluation of the communitybased adherence club model among newly postpartum women who had initiated ART during their recent pregnancy in Cape Town, South Africa. Our aim was to explore uptake of the adherence club model during the postpartum period, as well as programmatic outcomes among women choosing to attend these clubs.

\section{Methods \\ Design and setting}

Conducted between February 2015 and October 2016, the Postpartum Adherence Clubs to Enhance Support (PACER) study (ClinicalTrials.gov NCT02417675) was a component of the MCH-ART study (NCT01933477), a multicomponent implementation science study evaluating strategies for delivering HIV care and treatment services during pregnancy and the postpartum period [21]. The aim of the PACER study was to pilot and evaluate the enrollment of postpartum women into a network of adherence clubs for receipt of ART care. The study took place around the Midwife-Obstetric Unit of the Gugulethu Community Health Centre, a setting characterized by high levels of poverty and a high antenatal HIV prevalence [21]. In this setting, Option $\mathrm{B}+$ guidelines were rolled out during 2013. All participants provided written informed consent prior to enrollment, and the 
study was approved by the Human Research Ethics Committee of the University of Cape Town's Faculty of Health Sciences as well as the Institutional Review Board of the Columbia University Medical Center.

\section{Parent MCH-ART study}

The design and results of the parent $\mathrm{MCH}-\mathrm{ART}$ study have been previously described [21, 22]. The study included a randomized trial of postpartum ART services, comparing (i) an integrated postnatal care service within the $\mathrm{MCH}$ setting for HIV-infected mothers and their infants for the duration of breastfeeding to (ii) the local standard of care, immediate referral to adult ART services for mothers and separate 'well baby' services for infants. The primary outcome was a combined endpoint of maternal engagement in HIV care and viral suppression (VS) $<50$ copies/mL at 12 months postpartum.

\section{PACER study methods}

The methods of the PACER study parallel those of the $\mathrm{MCH}-\mathrm{ART}$ study, although separate cohorts were enrolled for each study. The PACER study methods and preliminary findings through 6 months postpartum have been reported previously [23]. Briefly, HIV-infected recently postpartum women who were $>18$ years old, had initiated ART during their recent pregnancy, intended to stay in the area through 12 months postpartum and were currently breastfeeding were eligible. In addition, women had to meet eligibility requirements for referral to the adherence club system: documented VS $<1000$ copies/ $\mathrm{mL}$ (based on VL at 12 weeks post ART initiation) and no comorbidities requiring on-going clinical review. Once enrolled into the PACER study, participants were given a choice between two options for their postpartum ART care: (i) referral to existing network of communitybased adherence clubs or (ii) referral to a local primary health care clinic (PHC). Following their choice, women were referred to their chosen service using standard referral procedures.

\section{Postpartum care services}

Antenatal services and the postpartum care services to which participants were referred have been previously described [23]. In brief, postpartum women were referred $<2$ weeks post-delivery from integrated antenatal ART/PMTCT services to a network of PHCs under the standard of care. PHCs provided general adult ART services at visits conducted every 1-2 months that included ART collection and a clinical consultation with a doctor or nurse. HIV-exposed infants were referred separately for routine 'well baby' services including early infant diagnosis (EID).

Community-based adherence clubs were facilitated by lay counselors and housed within community venues separate from public sector health facilities. Clubs met every $2-4$ months at which time patients were weighed, completed a brief symptom questionnaire and participated in health education sessions. Pre-packed ART was dispensed at all visits to patients or "treatment buddies" identified by the patient. Clinical review and VL testing were conducted annually by a nurse. Patients were referred back to routine PHC if they missed a scheduled club visit, had a detectable VL or comorbidity requiring clinical management, based on the standard criteria for continued attendance at clubs in this setting.

\section{Study follow-up \& sources of data}

Regardless of their choice of postpartum service, all women enrolled in the PACER study were followed at repeated study measurement visits that were scheduled and located separately from any club or PHC care visits through 12 months postpartum. These study measurement visits were held within a dedicated research center and conducted by trained study staff. At each study visit, questionnaires related to demographics, $\mathrm{MCH}$ service utilization, ART adherence, and breastfeeding practices were administered. Questions regarding ART service experience were asked at 9 months postpartum. Additionally, women provided $5 \mathrm{ml}$ of venous blood at each visit for batched HIV RNA VL testing by the South African National Health Laboratory Services (NHLS) using the Abbott RealTime HIV-1 assay (Abbott Laboratories, Abbott Park, Illinois, US).

Data on maternal and infant use of healthcare services were drawn from routinely collected public sector medical records (including facility registers, electronic medical records and infants' Road to Health Booklets) and centralized NHLS databases of laboratory tests, abstracted for all participants at the end of the study period.

\section{Outcomes}

The primary study outcome was a composite endpoint of women's retention in postpartum ART services and $\mathrm{VS}<50$ copies/mL (based on VL testing at measurement visits) at 12 months postpartum. Women were required to be both retained in care and virally suppressed in order to be considered as having achieved the primary outcome. Retention in care at 12 months postpartum was measured using routinely collected medical records and defined as evidence of an HIV-related clinical contact (whether from HIV-related laboratory testing or clinical care/adherence club visit) from 9 to 18 months postpartum. Where women had evidence of multiple clinical contacts from 9 to 18 months postpartum, we used the contact closest to 12 months postpartum to classify women as retained in a PHC service versus retained in an adherence club. Secondary outcomes 
included duration of any breastfeeding and exclusive breastfeeding (EBF) (self-reported at all study visits), and infant engagement outcomes (evidence of PCR testing for EID and immunizations) abstracted from routinelycollected medical records. Because outcome data on retention in postpartum ART care came from medical records available from all facilities in the province and did not require separate study follow-up, this outcome was available for all participants (regardless of completion of study visits and availability of VL outcome data, or movement out of Gugulethu) and is presented for all women enrolled as well as restricted to women who had VL outcome data.

\section{Analysis}

Data were analysed using Stata 12 (StataCorp Inc., College Station, Texas, USA). Variables were described using medians (with interquartile ranges, IQR) and proportions. We examined factors associated with retention at study measurement visits (defined as attending a study visit $>270$ days postpartum) using Wilcoxon ranksum (Mann Whitney) and chi-square or Fisher's exact tests in the case of sparse data. We compared primary and secondary study outcomes across postpartum ART service choice using chi-square and Fisher's exact tests, and used product-limit methods and the log-rank test to compare duration of breastfeeding and EBF across postpartum ART service choice. For the primary outcome, we defined VS as < 50 copies/mL, but examined VS < $1000 \mathrm{copies} / \mathrm{mL}$ in sensitivity analyses. In additional sensitivity analyses, we explored the effect of loss to followup by assuming that women who were not retained at study measurement visits had (i) suppressed and (ii) elevated VL, respectively. In order to account for potential confounding, we examined the independent effect of choice of ART service on the primary outcome in Poisson regression models with robust error variance. Given that our intention was to assess programmatic outcomes among women choosing to attend adherence clubs, we additionally explored referral out of clubs and compared the primary outcome across the service where women were receiving care at 12 months postpartum.

\section{Results}

\section{Study enrollment and retention}

A total of 129 women (median age: 28.9 years; median time postpartum: 10 days) were enrolled between February and September 2015 (Table 1). Women had been on ART for a median time of 23.3 weeks at enrollment, $80 \%$ had experienced a previous pregnancy, and most (59\%) were newly diagnosed HIV-positive during their recent pregnancy. A total of 84 women (65\%) opted to receive postpartum HIV care through an adherence club, with no differences in demographic or clinical characteristics among women choosing adherence clubs versus those choosing PHC. A total of 110 women (85\%) were retained at study measurement visits through 270 days postpartum, with a median time at outcome assessment of 12.1 months postpartum (IQR: 12.0-12.3 months). Compared to women retained at study measurement visits, women lost to follow-up were significantly more likely to have opted to receive postpartum HIV care from a PHC (63\% versus $37 \%$; $p=0.005)$.

\section{Comparison of primary outcome across ART services}

Among the 110 women retained at study measurement visits, 91 (83\%) achieved the composite endpoint of engagement in care and VS at 12 months postpartum, with no difference between those who originally chose adherence clubs versus those who chose PHC services $(84 \%$ versus $79 \%$, respectively; $p=0.583$; Table 2 ). Within this composite outcome, $87 \%$ of women $(n=96)$ had evidence of retention in ART services at 12 months postpartum, with retention in care not significantly different by choice of ART service: $90 \%$ of those who originally chose club services were retained in care, compared to $82 \%$ of those who chose PHC services $(p=0.349)$. Among all participants enrolled $(n=129)$, retention at study measurement visits was not associated with retention in care $(p=0.304)$. In this total sample, retention in care outcomes were similar across choice of ART services: $88 \%$ of women who chose club services were retained in care, compared to $82 \%$ of women who chose PHC services $(p=0.426)$. Similarly, no difference was observed in VS by ART service under various definitions of viral suppression (Table 2). For example, 90\% of women who chose adherence club services and $85 \%$ of women who chose PHC services had VS $<1000$ copies $/ \mathrm{mL}$ at 12 months postpartum $(p=0.525)$. After adjustment for maternal age and duration on ART, the lack of association between original choice of postpartum ART service and VS $<50$ copies $/ \mathrm{mL}$ at 12 months postpartum persisted (risk ratio for adherence club versus PHC: 1.08; 95\% confidence interval: $0.88,1.31$ ).

Given loss to follow-up from the study and assuming that women who were not retained at study measurement visits had (i) suppressed and (ii) elevated VL, respectively, the proportion of women with VS $<50$ copies $/ \mathrm{mL}$ at 12 months postpartum in the total sample ranged between 71 and $85 \%$. Under the assumption that all women lost from study measurement visits had suppressed VL $<50$ copies/mL, no difference between those who originally chose adherence clubs versus those who chose PHC services was observed ( $86 \%$ versus $84 \%$, respectively; $p=1.000$ ). However, when all women lost to follow-up were assumed to have elevated VL, those who originally chose adherence clubs were significantly more 
Table 1 Demographic and clinical characteristics of participants at enrolment, stratified by retention at study visits

\begin{tabular}{|c|c|c|c|c|}
\hline Variable & $\begin{array}{l}\text { Total sample - } \\
n(\%)\end{array}$ & $\begin{array}{l}\text { Completed study visit }>270 \text { days } \\
\text { postpartum }-n(\%)\end{array}$ & $\begin{array}{l}\text { Lost to study } \leq 270 \text { days } \\
\text { postpartum }-n(\%)\end{array}$ & $\begin{array}{l}p- \\
\text { value }\end{array}$ \\
\hline Number of women & 129 & 110 & 19 & \\
\hline \multicolumn{5}{|l|}{ ART service choice } \\
\hline Adherence club & $84(65)$ & $77(70)$ & $7(37)$ & \\
\hline Primary care clinic & $45(35)$ & $33(30)$ & $12(63)$ & 0.005 \\
\hline Median [IQR] age & $28.9[24.5,32.1]$ & $28.8[24.3,32.3]$ & $28.9[25.6,31.2]$ & 0.984 \\
\hline First pregnancy & $26(20)$ & $24(22)$ & $2(11)$ & 0.360 \\
\hline $\begin{array}{l}\text { Completed secondary/any tertiary } \\
\text { education }\end{array}$ & $60(47)$ & $53(48)$ & $7(37)$ & 0.360 \\
\hline Currently employed & $45(35)$ & $40(36)$ & $5(26)$ & 0.396 \\
\hline Married/cohabiting & $48(37)$ & $41(37)$ & $7(37)$ & 0.971 \\
\hline Newly diagnosed HIV+ in this pregnancy & $76(59)$ & $67(61)$ & $9(47)$ & 0.268 \\
\hline \multicolumn{5}{|l|}{ Pregnancy intention } \\
\hline Unintended & $88(68)$ & $74(67)$ & $14(74)$ & 0.790 \\
\hline Intended & $41(32)$ & $36(33)$ & $5(26)$ & \\
\hline \multicolumn{5}{|l|}{ Median [IQR] CD4 cell count } \\
\hline$\leq 350$ cells $/ \mu \mathrm{L}$ & $56(43)$ & $48(44)$ & $8(42)$ & \\
\hline$>350$ cells $/ \mu \mathrm{L}$ & $73(57)$ & $62(56)$ & $11(58)$ & 0.901 \\
\hline \multicolumn{5}{|l|}{ HIV viral load } \\
\hline$<50$ copies $/ \mathrm{mL}$ & $114(93)$ & $99(94)$ & $15(83)$ & \\
\hline$\geq 50$ copies $/ \mathrm{mL}$ & $9(7)$ & $6(6)$ & $3(17)$ & 0.126 \\
\hline Median [IQR] time on ART (weeks) & $23.3[18.1,26.9]$ & $23.7[18.4,27.0]$ & $21.0[15.6,26.7]$ & 0.266 \\
\hline Median [IQR] days postpartum & $10[5,19]$ & $10[6,19]$ & $8[5,15]$ & 0.180 \\
\hline \multicolumn{5}{|l|}{ Place of delivery } \\
\hline Primary care & $50(39)$ & $41(37)$ & $9(47)$ & \\
\hline Hospital care & $79(61)$ & $69(63)$ & $10(53)$ & 0.404 \\
\hline $\begin{array}{l}\text { Reported missed ART dose(s) during } \\
\text { previous } 30 \text { days }\end{array}$ & $19(15)$ & $18(16)$ & $1(5)$ & 0.304 \\
\hline $\begin{array}{l}\text { Exclusively breastfed infant up to } \\
\text { enrolment }\end{array}$ & $106(82)$ & $88(80)$ & $18(95)$ & 0.193 \\
\hline
\end{tabular}

likely to have suppressed VL compared to those who chose PHC services (77\% versus 58\%, respectively; $p=$ 0.026).

\section{Comparison of secondary outcomes across ART services}

The median duration of any breastfeeding was 3.2 months [IQR: 1.4-11.8 months] and did not differ by choice of postpartum ART service (Fig. 1a). Among 106 women who reported EBF at enrolment, the median duration of EBF was 3.0 months [IQR: 1.2-3.1 months (Fig. 1b). Uptake of infant care services was high in both groups. Overall, 95\% of mothers had evidence of infant PCR testing between birth and 12 weeks of age (Table 2). Among 106 women who were retained in the study with infant medical records available, uptake of infant immunization through 10 weeks of infant age was similarly high. Neither breastfeeding nor uptake of infant care services differed across ART service choice.

\section{Experiences in postpartum ART services}

Of the 110 women retained at study measurement visits, 102 completed the ART service experience assessment at 9 months postpartum. Women attending an adherence club were positive about these services, with $98 \%$ reporting that services were "excellent" or "very good" (Table 3). Participants cited support from peers and counsellors, less frequent and shorter visits, and receiving care outside of health facilities as aspects of club services that they liked. In contrast, women attending PHC services reported mixed feelings about these services, with $65 \%$ reporting that the services were "excellent" or "very good". These women were also less positive about the frequency and duration of visits, although $78 \%$ 
Table 2 Comparison of composite endpoint and uptake of infant care services by ART service choice

\begin{tabular}{|c|c|c|c|c|}
\hline Variable & $\begin{array}{l}\text { Total sample } \\
- \\
n(\%)\end{array}$ & $\begin{array}{l}\text { Adherence club } \\
- \\
n(\%)\end{array}$ & $\begin{array}{l}\text { Primary care clinic } \\
- \\
n(\%)\end{array}$ & $\begin{array}{l}p- \\
\text { value }\end{array}$ \\
\hline \multicolumn{5}{|c|}{ Composite endpoint: Evidence of maternal retention in HIV care AND VL $<50$ copies $/ \mathrm{mL}$ at 12 months postpartum } \\
\hline Number of women eligible for composite endpoint & 110 & 77 & 33 & \\
\hline N (\%) achieving composite endpoint & $91(83)$ & $65(84)$ & $26(79)$ & 0.583 \\
\hline \multicolumn{5}{|c|}{ Retention in care (in all participants retained at study visits with VL data available) } \\
\hline Number of women retained at study visits with VL data available & 110 & 77 & 33 & \\
\hline Evidence of engagement in HIV care at 12 months postpartum & $96(87)$ & $69(90)$ & $27(82)$ & 0.349 \\
\hline \multicolumn{5}{|l|}{ Retention in care (in all participants enrolled) } \\
\hline Number of women enrolled & 129 & 84 & 45 & \\
\hline Evidence of engagement in HIV care at 12 months postpartum & $111(86)$ & $74(88)$ & $37(82)$ & 0.426 \\
\hline \multicolumn{5}{|l|}{ Viral load (in all participants retained at study visits with VL data available) } \\
\hline Number of women retained at study visits with VL data available & 110 & 77 & 33 & \\
\hline $\mathrm{VL}<50$ copies $/ \mathrm{mL}$ at 12 months postpartum & $91(83)$ & $65(84)$ & $26(79)$ & 0.583 \\
\hline$V L<1000$ copies/mL at 12 months postpartum & $97(88)$ & $69(90)$ & $28(85)$ & 0.525 \\
\hline \multicolumn{5}{|l|}{ Infant care services } \\
\hline Number of women & 129 & 84 & 45 & \\
\hline Evidence of infant PCR test $<12$ weeks of age & $123(95)$ & $81(96)$ & $42(93)$ & 0.420 \\
\hline $\begin{array}{l}\text { Number of women retained in the study with infant immunization records } \\
\text { available }\end{array}$ & 106 & 75 & 31 & \\
\hline Evidence of birth immunizations & $104(98)$ & $73(97)$ & $31(100)$ & 1.000 \\
\hline Evidence of 6 week immunizations & $99(93)$ & $68(91)$ & $31(100)$ & 0.103 \\
\hline Evidence of 10 week immunizations & $97(92)$ & $68(91)$ & $29(94)$ & 1.000 \\
\hline
\end{tabular}

VL Viral load

reported that they preferred to receive care within a health facility.

\section{Comparison with the parent MCH-ART study}

A total of 471 women were enrolled into the randomized trial of the parent MCH-ART study, with women randomized to the integrated $\mathrm{MCH}$-focused postpartum care intervention having significantly higher levels of retention and VS through 12 months postpartum compared to women randomized to PHC services [22]. When comparing the $\mathrm{PHC}$ arms of the parent $\mathrm{MCH}-$ ART and the PACER study, we observed higher levels of engagement in care and VS at 12 months postpartum among women accessing standard of care PHC services in the PACER study: 79\% of PACER participants choosing $\mathrm{PHC}$ services achieved the composite endpoint versus $56 \%$ of participants randomized to PHC services in the parent study. However, levels of engagement and VS were higher among women randomized to the $\mathrm{MCH}$ focused service in the parent study (84\%) versus either the MCH-ART or PACER PHC arm. Duration of breastfeeding was similar across women choosing club services and those attending PHC services in both cohorts, but was significantly longer in women attending the $\mathrm{MCH}$ - focused service in the parent study (Fig. 1a and b). No appreciable differences in the uptake of infant care services were observed across any of the ART services.

\section{Movement between postpartum ART services}

Movement from adherence clubs to PHC services is described in Fig. 2. Of the 84 women who originally chose to attend adherence club services, 13 (15\%) were found to have never attended their first club meeting [21]. On further medical record review, 12 of these women were found to have immediately accessed care at a PHC after postpartum referral, and 2 later joined an adherence club. Among the 74 women who originally chose to attend club services and were engaged in care at 12 months postpartum, 31\% $(n=23)$ were found to be engaged at a PHC service; 2 women (5\%) who originally chose PHC services and were engaged in care at 12 months postpartum had evidence of engagement at an adherence club.

Given high levels of movement from adherence clubs back to PHC services, we explored VS outcomes by the location of ART care. Among the 96 women who had evidence of retention in ART services at 12 months postpartum and VL data available, levels of VS differed 

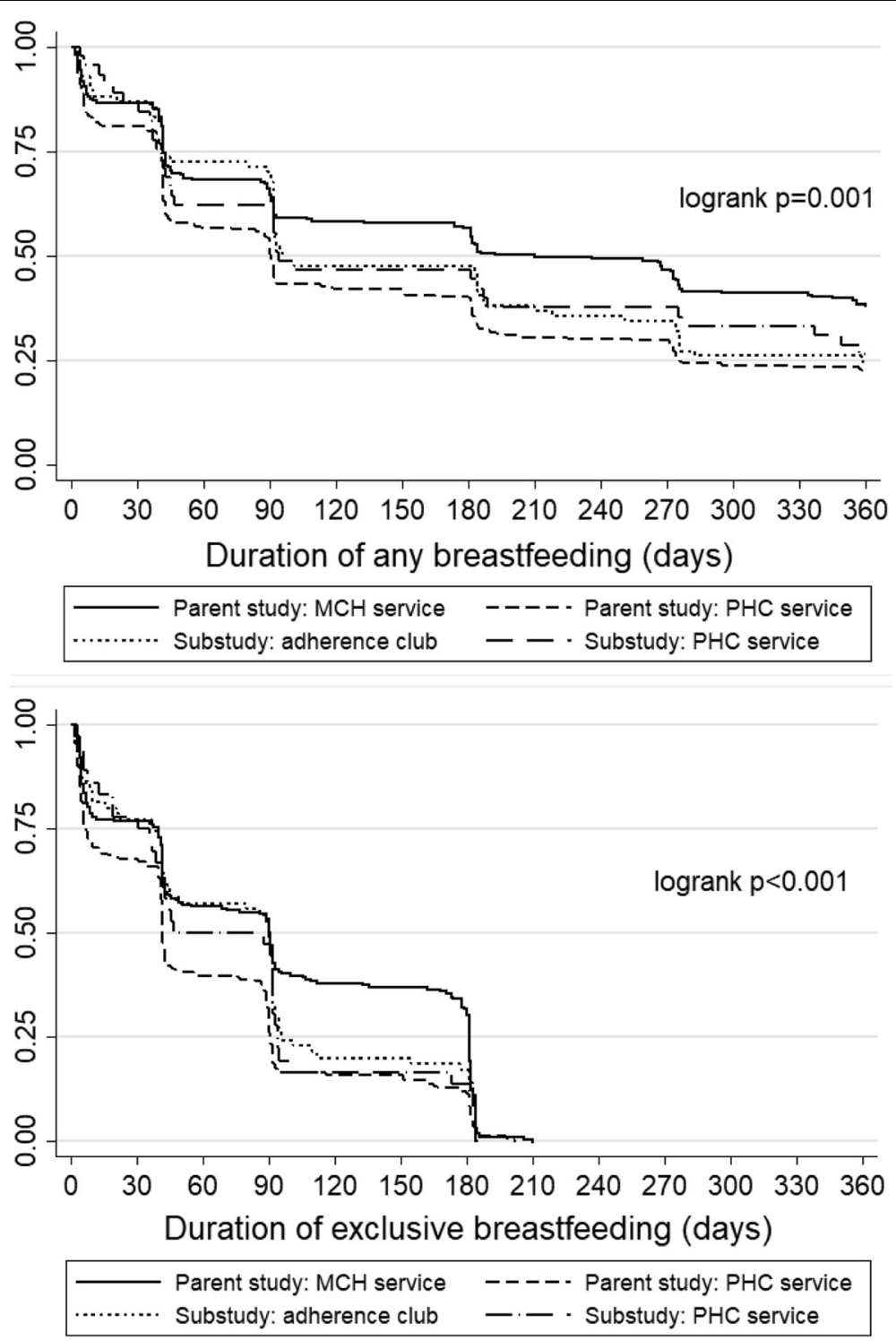

Fig. 1 a Time to cessation of any breastfeeding, stratified by postpartum ART service [maternal/child health (MCH) service, primary health care (PHC) service, or adherence club]. b Time to cessation of exclusive breastfeeding, stratified by postpartum ART service [maternal/child health $(\mathrm{MCH})$ service, primary health care $(\mathrm{PHC})$ service, or adherence club]

significantly across the location of ART care: $98 \%$ of those receiving care in an adherence club and $76 \%$ of women receiving care at PHC services had VS $<50$ copies $/ \mathrm{mL}$ at 12 months postpartum $(p=0.001)$, regardless of initial choice of postpartum ART services. The results of VL testing at study measurement visits are shown in Fig. 3, by original choice of ART service and location of ART care at 12 months postpartum. Overall, women who chose to attend adherence clubs and remained in these services through 12 months postpartum were less likely to experience elevated VL compared to women who chose to attend adherence clubs but later moved to PHC services, or women who chose to attend PHC services and stayed in these services through 12 months postpartum.

\section{Discussion}

In this study of participation in adherence clubs among newly postpartum women, we found comparable outcomes related to retention in care and VS at 12 months postpartum between women choosing adherence clubs and those choosing PHC services. These data provide longer-term virologic outcomes that are consistent with and compliment the short-term (6-months postpartum) VS outcomes previously reported [23]. While the earlier short-term outcomes captured the immediate postnatal 
Table 3 Experiences in ART services $(n=102)$

\begin{tabular}{|c|c|c|c|}
\hline Adherence club $(n=65)$ & $n(\%)$ & Primary care clinic (PHC; $n=37)$ & $n(\%)$ \\
\hline Number of visits at adherence club & & Number of visits at $\mathrm{PHC}^{\mathrm{a}}$ & \\
\hline 1 & $6(9)$ & 1 & $2(5)$ \\
\hline 2 & $27(42)$ & 2 & $12(32)$ \\
\hline 3 or more & $32(49)$ & 3 or more & $22(59)$ \\
\hline Prefers that club is closer to where she lives compared to PHC & $47(72)$ & Prefers that PHC is closer to where she lives compared to club & $24(67)$ \\
\hline Likes that club is close to where her baby receives healthcare & $45(69)$ & Likes that PHC is close to where her baby receives healthcare & $24(65)$ \\
\hline Prefers to receive care outside of health facility & $61(94)$ & Prefers to receive care within health facility & $29(78)$ \\
\hline Likes that there are only 5 visits per year & $63(97)$ & Likes having visits every $4-8$ weeks & $21(58)$ \\
\hline Likes that the visits are $1 \mathrm{~h}$ or less & $62(95)$ & Likes the amount of time spent at the PHC and pharmacy & $16(44)$ \\
\hline Likes not seeing a doctor/nurse frequently & $51(78)$ & Likes seeing a doctor/nurse frequently & $25(68)$ \\
\hline Likes receiving peer support from club group & $64(98)$ & - & - \\
\hline Likes receiving care and support from a counsellor & $64(98)$ & - & - \\
\hline Overall feelings about club & & Overall feelings about PHC & \\
\hline Excellent & $38(58)$ & Excellent & $9(24)$ \\
\hline Very good & $26(40)$ & Very good & $15(41)$ \\
\hline Good & $1(2)$ & Good & $6(16)$ \\
\hline Bad & $0(0)$ & Bad & $4(11)$ \\
\hline Very bad & $0(0)$ & Very bad & $3(8)$ \\
\hline
\end{tabular}

${ }^{\mathrm{a} M i s s i n g}$ data from one participant

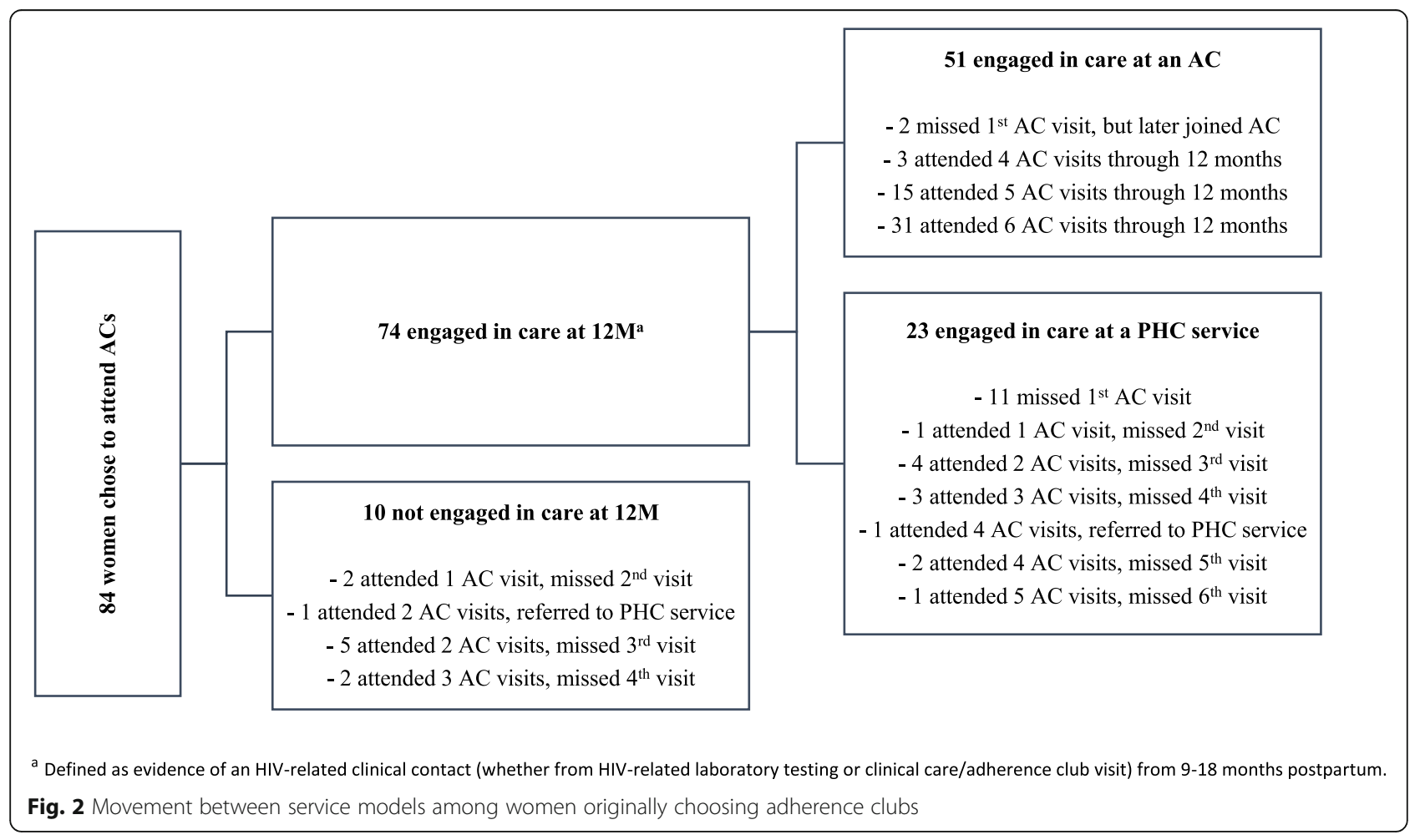




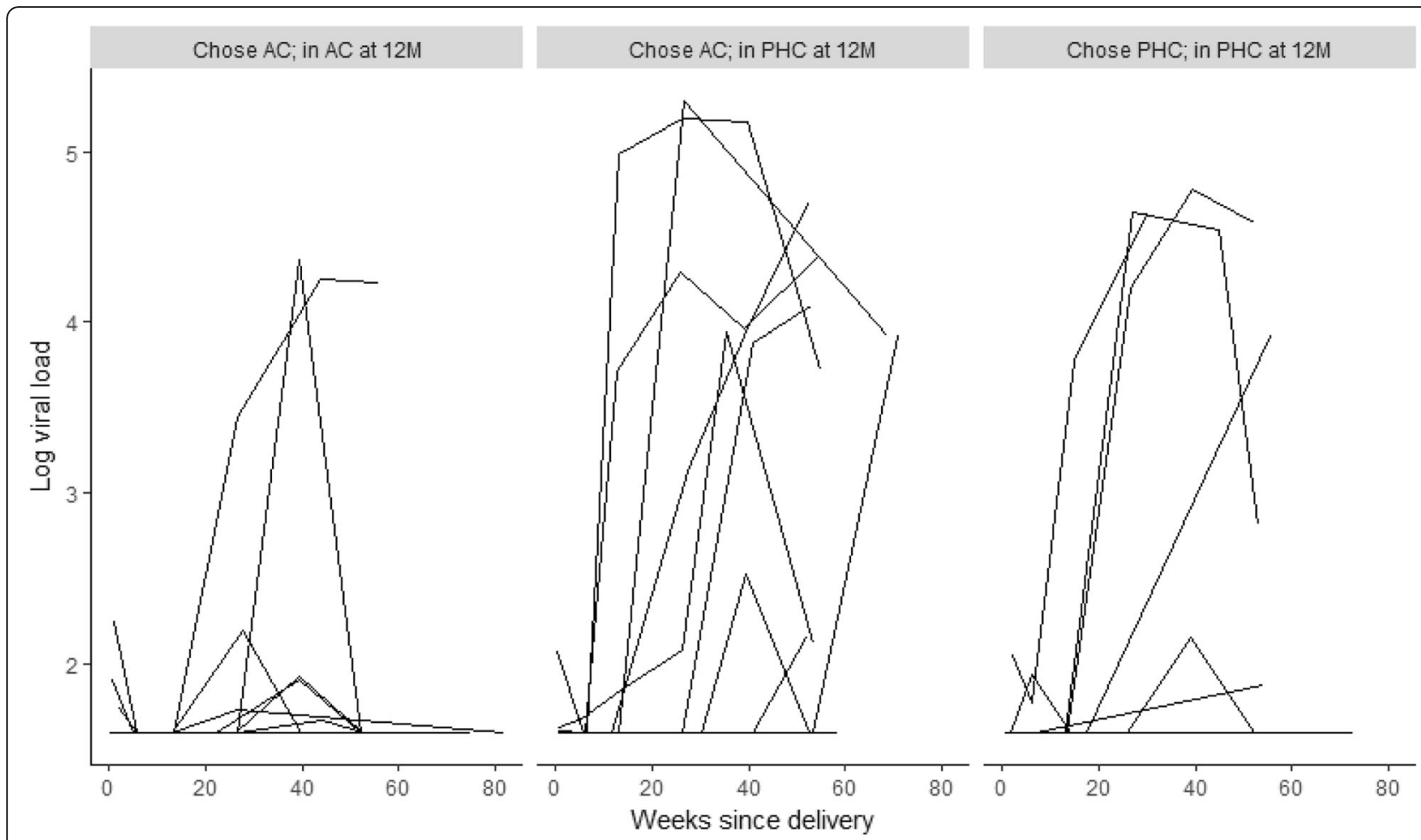

Fig. 3 Spaghetti plots of viral load measures conducted at study measurement visits, from delivery through approximately 12 months postpartum, with each line representing a participant and stratified by women who 1) originally chose to attend adherence clubs (AC) and remained in AC at 12 months postpartum (left-hand panel), 2) originally chose to attend AC but moved to primary health care (PHC) services by 12 months postpartum (center panel) and 3) originally chose to attend PHC and remained in PHC at 12 month postpartum

period- a critical time in the PMTCT cascade- these longer-term outcomes are important given the welldocumented challenges around non-adherence and disengagement from care among this group [7]. Despite the high overall levels of engagement and VS observed here, we note substantial movement out of adherence clubs and significantly poorer VS outcomes among women who leave adherence clubs and return to PHC services. Regarding secondary outcomes, postpartum women attending adherence clubs were comparable to those attending PHCs in terms of duration of breastfeeding and uptake of infant care services.

Women who chose and attended clubs reported greater satisfaction with these services than those who opted for and attended PHCs. Despite this, we found a significant amount of movement between postpartum services among those who originally chose adherence clubs. By the end of the study follow-up period, one third of those who initially chose and attended the clubs and were engaged in care at 12 months postpartum were found to be attending a PHC. The finding that these women have significantly poorer VS outcomes compared to those who originally chose and remained in adherence clubs suggests that women who remain in clubs do well in this service and that those who leave the service (either by choice or referral back due to clinical indication) and return to PHC services have poorer VS outcomes, despite remaining engaged in care.

These data also highlight the complexities of monitoring and evaluating the impact of adherence clubs and DSD models, generally. We have previously discussed how evaluations that begin with patients making their first visit to an adherence club likely overestimate retention, given the high proportion of women who did not attend their first adherence club visit [23]. Here, we demonstrate that a cross-sectional analysis of women attending adherence clubs at 12 months postpartum does not consider those who have dropped out of clubs or who were referred back to PHC services and who by definition have poor outcomes. As women who newly initiated ART during pregnancy, none of the participants had experience with either postpartum service at the time that they were asked to choose a location for their postpartum care. It is possible that upon attending their chosen service, other factors (geography, fear of stigma, desire for more clinical oversight) emerged and influenced their decision to remain in the club or to seek care elsewhere [24, 25]. High levels of mobility among postpartum women, including in South Africa, have been documented and may also play a role in patterns of 
clinic movement $[26,27]$. There are specific vulnerabilities associated with postpartum transition from one service delivery model to another and increased risk associated with this transfer process [25]. Interventions to facilitate and monitor the safe transition from one model or site to another is an issue that requires further attention as this DSD model is expanded.

The duration of breastfeeding here was comparable among postpartum women attending clubs and the PHC arm of both the parent MCH-ART study and PACER study but was significantly longer in women attending the $\mathrm{MCH}$-focused service in the parent study. Unlike the intervention arm of the MCH-ART study, women in the PACER study were referred to a network of existing adherence clubs, none of which were tailored or customized to the needs of postpartum women. The need for multilevel support to address the multiple and complex needs of postpartum women and their families during this time is of critical importance. Integration of $\mathrm{MCH}$ services including family planning, counselling around infant feeding and HIV PCR testing for infants into clubs specifically for postpartum women is an idea that has not yet been explored fully, may be critical to the success of DSD for this population and should be considered as the AC model expands both in South Arica and other settings. An additional consideration may also include whether these groups could be further tailored for women with known HIV infection and who are stable on ART at the time of entry into ANC services versus those who are newly diagnosed and initiated ART in their most recent pregnancy [28].

These results are subject to several limitations. For this pilot study, women were offered a choice of postpartum ART services. Given that women were not randomly allocated to services, confounding may be a concern, but we observed no differences in demographic or clinical characteristics between women choosing each service, and adjustment for age and duration on ART did not change the results observed. Further, exposure to the intervention (postpartum service) changed over time as women moved from adherence clubs to PHCs. However, it was not possible to accurately parse out periods during which women were in the adherence clubs versus PHCs versus not in care, as not all data systems include scheduled appointment dates. The setting for this study was limited to adult women from one urban area in South Africa and results may not be generalizable to other populations. The study sample included only women who had initiated ART during their recent pregnancy, thus there was little variability in the duration of ART use among these women. However, future studies should evaluate the effectiveness of postpartum adherence clubs for women who are already established on ART when entering antenatal care. Additionally, while this is the first evaluation of its kind, the sample size was relatively small and thus the power to detect quantitative associations is limited. In total, $15 \%$ of women were lost from study measurement visits, which is consistent with the amount of loss to follow-up in the parent $\mathrm{MCH}$ ART study (13\%) [22]. This study is strengthened by its use of VL measures carried out independently from routine care and a retention measure that was not specific to only the facility of interest and was available for all women regardless of retention at study measurement visits. In addition, outcomes included retention and VS measured over a 12-month period. We observed high levels of retention in care and VS overall, likely due to the fact that all women enrolling into the study were required to meet the eligibility criteria for referral into the adherence club system, including VS $<1000$ copies $/ \mathrm{mL}$. However, under the assumption that all women lost from study measurement visits had elevated VL at 12 months postpartum, the proportion with VS was lower ( $71 \%$ versus $83 \%$ when restricted to women retained in the study).

\section{Conclusions}

In summary, this study suggests comparable outcomes related to retention in care and VS at 12 months postpartum between women choosing adherence clubs and those choosing PHC services. We observed a higher reported degree of satisfaction among women attending adherence clubs, but this did not translate into higher rates of retention in the club, with many women leaving the clubs to return to the standard of care facilities, and higher levels of elevated VL in these women. Ongoing work to understand adherence and retention among postpartum women is needed given the growing number of women on ART in the postpartum period. To optimize implementation of adherence clubs and other DSD models among newly postpartum women, additional work is needed to identify strategies to integrate $\mathrm{MCH}$ services into these settings.

\section{Abbreviations}

ART: Antiretroviral therapy; DSD: Differentiated service delivery; EBF: Exclusive breastfeeding; EID: Early infant diagnosis; IQR: Interquartile range;

MCH: Maternal child health; PHC: Primacy health clinic; PMTCT: Prevention of mother-to-child transmission; VL: Viral load; VS: Viral suppression; WHO: World Health Organization

\section{Acknowledgements \\ The authors thank the study team for their support and contribution to the study. The authors are also grateful to the study participants included in this} study.

\section{Authors' contributions}

AZ developed data collection instruments, assisted with supervision of research activities and wrote the manuscript. KB led data analysis and contributed to manuscript development. TP developed data collection instruments, supervised research activities and data collection, contributed to data analysis, and contributed to manuscript development. VI supervised research activities and data collection and contributed to manuscript 
development. JO contributed to research activities, contributed to data analysis and contributed to manuscript development. AN contributed to research activities. CK contributed to research activities and contributed to manuscript development. LM conceived the study, assisted with development of data collection instruments, assisted with supervision of research activities and contributed to manuscript development. EJA conceived the study, assisted with development of data collection instruments, assisted with supervision of research activities, contributed to manuscript development. All authors have read and approved the final version.

\section{Funding}

This research was supported by the President's Emergency Plan for AIDS Relief (PEPFAR) through the National Institute of Child Health and Human Development (NICHD), Grant Number 1R01HD074558. Additional funding comes from the Elizabeth Glaser Pediatric AIDS Foundation. Study funders were not directly engaged with the study and had no contact with study participants and no access to individually identifiable private information.

\section{Availability of data and materials}

The datasets used and/or analyzed during the current study are available from the corresponding author on reasonable request.

\section{Ethics approval and consent to participate}

All participants provided written informed consent prior to enrollment, and the study was approved by the Human Research Ethics Committee of the University of Cape Town's Faculty of Health Sciences as well as the Institutional Review Board of the Columbia University Medical Center.

\section{Consent for publication}

Not Applicable.

\section{Competing interests}

The authors declare that they have no competing interests.

\section{Author details}

'ICAP, Mailman School of Public Health, Columbia University, 722 W. 168th street, 13th floor, New York 10032, USA. ${ }^{2}$ Centre for Infectious Disease Epidemiology \& Research, School of Public Health \& Family Medicine, University of Cape Town, Cape Town, South Africa. ${ }^{3}$ Division of Epidemiology \& Biostatistics, School of Public Health \& Family Medicine, University of Cape Town, Cape Town, South Africa. ${ }^{4}$ Provincial Government of the Western Cape, Cape Town, South Africa. ${ }^{5}$ Vagelos College of Physicians \& Surgeons, Columbia University, New York, USA.

Received: 4 February 2019 Accepted: 25 June 2020

Published online: 08 July 2020

\section{References}

1. UNAIDS. Global AIDS Update. Geneva: UNAIDS; 2016

2. Haas AD, Tenthani L, Msukwa MT, Tal K, Jahn A, Gadabu OJ, et al. Retention in care during the first 3years of antiretroviral therapy for women in Malawi's option B+ programme: an observational cohort study. Lancet HIV 2016:3(4):e175-82

3. Miller K, Muyindike W, Matthews LT, Kanyesigye M, Siedner MJ. Program implementation of option B+ at a President's emergency plan for AIDS relief-supported HIV clinic improves clinical indicators but not retention in Care in Mbarara, Uganda. AIDS Patient Care STDS. 2017:31(8):335-41.

4. Pfeiffer JT, Napúa M, Wagenaar BH, Chale F, Hoek R, Micek M, et al. A stepped wedge cluster randomized controlled trial to promote Option B+ retention in central Mozambique. J Acquir Immune Defic Syndr. 2017;76(3): 273-80

5. Etoori D, Kerschberger B, Staderini N, Ndlangamandla M, Nhlabatsi B, Jobanputra K, Mthethwa-Hleza S, Parker LA, Sibanda S, Mabhena E, Pasipamire M, Kabore SM, Rusch B, Jamet C, Ciglenecki I, Teck R. Challenges and successes in the implementation of option B+ to prevent mother-tochild transmission of HIV in southern Swaziland. BMC Public Health. 2018. 18(1):374.

6. Abrams EJ, Langwenya N, Gachuhi A, Zerbe A, Nuwagaba-Biribonwoha H, Mthethwa-Hleta S, et al. Impact of universal antiretroviral therapy for pregnant and postpartum women on antiretroviral therapy uptake and retention. AIDS. 2019:33:45-54

7. Knettel BA, Cichowitz C, Ngocho JS, Chumba LN, Mmbaga BT, et al. Retention in HIV care during pregnancy and the postpartum period in the option B+ era: systematic review and meta-analysis of studies in Africa. J Acquir Immune Defic Syndr. 2018 Apr 15;77(5):427-38.

8. Bemelmans M, Baert S, Goemaere E, Wilkinson L, Vandendyck M, van Cutsem G, et al. Community- supported models of care for people on HIV treatment in sub-Saharan Africa. Trop Med Int Health. 2014;19(8): 968-77.

9. Okoboi S, Ding E, Persuad S, Wangisi J, Birungi J, Shurgold S, et al. Community-based ART distribution system can effectively facilitate longterm program retention and low-rates of death and virologic failure in rural Uganda. AIDS Res Ther. 2015;12:37.

10. Naslund JA, Dionne-Odom J, Junior Destiné C, Jogerst KM, Renold Sénécharles $R$, Jean Louis $M$, et al. Adapting and implementing a community program to improve retention in care among patients with HIV in southern Haiti: "group of 6". AIDS Res Treat. 2014;2014:137545.

11. Consolidated Guidelines on the Use of Antiretroviral Drugs for Treating and Preventing HIV Infection: Recommendations for a Public Health Approach. 2nd edition. Geneva: World Health Organization; 2016.

12. Decroo T, Rasschaert F, Telfer B, Remartinez D, Laga M, Ford N. Communitybased antiretroviral therapy programs can overcome barriers to retention of patients and decongest health services in sub-Saharan Africa: a systematic review. Int Health. 2013;5(3):169-79.

13. Wilkinson LS. ART adherence clubs: a long-term retention strategy for clinically stable patients receiving antiretroviral therapy. Southern Afr J HIV Med. 2013;14(2):48-50.

14. Luque-Fernandez MA, Van Cutsem G, Goemaere E, Hilderbrand K, Schomaker M, Mantangana N, et al. Effectiveness of patient adherence groups as a model of care for stable patients on antiretroviral therapy in Khayelitsha, Cape Town, South Africa. PLoS One. 2013;8(2):e56088.

15. Mdege ND, Chindove S, Ali S. The effectiveness and cost implications of task-shifting in the delivery of antiretroviral therapy to HIV-infected patients: a systematic review. Health Policy Plan. 2013;28(3):223-416 36.

16. Barker C, Dutta A, Klein K. Can differentiated care models solve the crisis in HIV treatment financing? Analysis of prospects for 38 countries in subSaharan Africa. J Int AIDS Soc. 2017;20(Suppl 4):21648.

17. Grimsrud A, Lesosky M, Kalombo C, Bekker LG, Myer L. Community-based adherence clubs for the Management of Stable Antiretroviral Therapy Patients in Cape Town, South Africa: a cohort study. J Acquir Immune Defic Syndr. 2016;71(1):e16-23.

18. Tsondai PR, Wilkinson LS, Grimsrud A, Mdlalo PT, Ullauri A, Boulle A. High rates of retention and viral suppression in the scale-up of antiretroviral therapy adherence clubs in Cape Town, South Africa. J Int AIDS Soc. 2017; 20(Suppl 4):21649

19. Srivastava M, Sullivan D, Phelps BR, Modi S, Broyles LN. Boosting ART uptake and retention among HIV-infected pregnant and breastfeeding women and their infants: the promise of innovative service delivery models. J Int AIDS Soc. 2018;21(1):e25053

20. World Health Organization. Key considerations for differentiated antiretroviral therapy delivery for specific populations: children, adolescents, pregnant and breastfeeding women and key populations. Geneva: World Health Organization; 2017.

21. Myer L, Phillips T, Zerbe A, Ronan A, Hsiao NY, Mellins CA, Remien RH, LeRoux SM, Brittain K, Ciaranello A, Petro G, Mclntyre JA, Abrams EJ. Optimizing antiretroviral therapy (ART) for maternal and child health $(M C H)$ : rationale and design of the MCH-ART study. J Acquir Immune Defic Syndr. 2016;72(Suppl2):S189-96.

22. Myer L, Phillips TK, Zerbe A, Brittain K, Lesosky M, Hsiao NY, Remien RH, Mellins CA, McIntyre JA, Abrams EJ. Integration of postpartum healthcare services for HIV-infected women and their infants in South Africa: a randomised controlled trial. PLoS Med. 2018;15(3):e1002547.

23. Myer L, Iyun V, Zerbe A, Phillips TK, Brittain K, Mukonda E, Allerton J, Kalombo CD, Nofemela A, Abrams EJ. Differentiated models of care for postpartum women on antiretroviral therapy in Cape Town, South Africa: a cohort study. J Int AIDS Soc. 2017;20(Suppl 4):32-40.

24. Clouse K, Schwartz S, Van Rie A, Bassett J, Yende N, Pettifor A. "What they wanted was to give birth; nothing else": barriers to retention in option B+ HIV care among postpartum women in South Africa. J Acquir Immune Defic Syndr. 2014;67(1):e12-8. 
25. Phillips TK, Clouse K, Zerbe A, Orrell C, Abrams EJ, Myer L. Linkage to care, mobility and retention of HIV-positive postpartum women in antiretroviral therapy services in South Africa. J Int AIDS Soc. 2018;21(Suppl 4):e25114.

26. Clouse K, Vermund SH, Maskew M, Lurie MN, MacLeod W, Malete G, Carmona S, Sherman G, Fox MP. Mobility and clinic switching among postpartum women considered lost to HIV Care in South Africa. J Acquir Immune Defic Syndr. 2017;74(4):383-9.

27. Ferguson $L$, Lewis J, Grant $A D$, et al. Patient attrition between diagnosis with HIV in pregnancy-related services and long-term HIV care and treatment services in Kenya: a retrospective study. J Acquir Immune Defic Syndr. 2012;60(3):e90-7.

28. Nelson AK, Cassidy T, Trivino Duran L, Hoxha A, Buchanan K, Shroufi A et al. Post natal clubs: A differentiated model of care integrating maternal, child health and prevention of mother to child transmission (PMTCT) in Khayelitsha, South Africa. [Abstract TTHPEE660] 22 ${ }^{\text {nd }}$ International AIDS Conference 23-27 July 2018.

\section{Publisher's Note}

Springer Nature remains neutral with regard to jurisdictional claims in published maps and institutional affiliations.

Ready to submit your research? Choose BMC and benefit from:

- fast, convenient online submission

- thorough peer review by experienced researchers in your field

- rapid publication on acceptance

- support for research data, including large and complex data types

- gold Open Access which fosters wider collaboration and increased citations

- maximum visibility for your research: over $100 \mathrm{M}$ website views per year

At BMC, research is always in progress.

Learn more biomedcentral.com/submissions 